\title{
IDEA: Intrinsic Dimension Estimation Algorithm
}

\author{
Alessandro Rozza, Gabriele Lombardi, Marco Rosa, \\ Elena Casiraghi, and Paola Campadelli \\ Dipartimento di Scienze dell'Informazione, Università degli Studi di Milano, \\ Via Comelico 39-41, 20135 Milano, Italy \\ rozza@dsi.unimi.it \\ http://security.dico.unimi.it/ fox721/
}

\begin{abstract}
The high dimensionality of some real life signals makes the usage of the most common signal processing and pattern recognition methods unfeasible. For this reason, in literature a great deal of research work has been devoted to the development of algorithms performing dimensionality reduction. To this aim, an useful help could be provided by the estimation of the intrinsic dimensionality of a given dataset, that is the minimum number of parameters needed to capture, and describe, all the information carried by the data. Although many techniques have been proposed, most of them fail in case of noisy data or when the intrinsic dimensionality is too high. In this paper we propose a local intrinsic dimension estimator exploiting the statistical properties of data neighborhoods. The algorithm evaluation on both synthetic and real datasets, and the comparison with state of the art algorithms, proves that the proposed technique is promising.
\end{abstract}

Keywords: Intrinsic dimension estimation, feature reduction, manifold learning.

\section{Introduction}

The high dimensionality of some real life signals, such as images, genome sequences, or EEG data, makes the usage of the most common signal processing and pattern recognition methods unfeasible. Nevertheless, many of these signals can be fully characterized by few degrees of freedom, represented by low dimensional feature vectors. In this case the feature vectors can be viewed as points constrained to lie on a low-dimensional manifold embedded in a higher dimensional space. The dimensionality of these manifolds is generally referred as intrinsic dimensionality. In more general terms, according to 8, a dataset is said to have intrinsic dimensionality equal to $d(1 \leq d \leq D)$ if its elements lie entirely within a $d$-dimensional subspace of $\Re^{D}$.

Intrinsic dimension estimation is important to discover structures, to perform dimensionality reduction and classification tasks. For this reason, in literature many techniques that estimate the intrinsic dimensionality have been proposed, which can be divided into two main groups: global approaches exploit the properties of the whole dataset to estimate the intrinsic dimensionality, whilst local approaches analyze the local behavior in the data neighborhoods. 
The most cited example of global method is the Principal Component Analysis (PCA, [12]). Since it is the easiest way to reduce dimensionality, provided that a linear dependence exists, it is often used to pre-process the data. To this aim, PCA projects the points on the directions of their maximum variance, estimated by performing the eigen-decomposition on the data covariance matrix. Exploiting PCA, $d$ can be estimated by counting the number of normalized eigenvalues that are higher than the threshold value $\rho$. Another interesting global approach is described in [3]; the authors exploit entropic graphs, such as the geodesic minimal spanning tree (GMST [2]) or the kNN-graph (kNNG), to estimate both the intrinsic dimensionality of a manifold, and the intrinsic entropy of the manifold random samples. Finally, we recall a well-known global intrinsic dimensionality estimator, which is the packing number method [13. Unfortunately, global approaches often fail dealing with non-linearly embedded manifolds or noisy data, and they are usually computationally too expensive on high dimensional datasets.

Local intrinsic dimensionality estimators, such as LLE [17, TVF [16], and Hessian eigenmaps [5], are based on properties related to neighboring points in the given dataset. Most of these techniques consider hyperspheres with sufficiently small radius and centered on the dataset points, and they estimate some statistics by considering the neighboring points, included into the hypersphere; these statistics are expressed as functions of the intrinsic dimension of the manifold from which the points have been drawn. One of these techniques is the Correlation Dimension (CD) estimator [9]; it is based on the assumption that the volume of a $d$-dimensional set scales with its size $r$ as $r^{d}$, which implies that also the number of samples covered by a hypersphere with radius $r$ grows proportionally to $r^{d}$. Another well known technique is the Maximum Likelihood Estimator (MLE) [15] (and its regularization [10]), that applies the principle of maximum likelihood to the distances between close neighbors, and derive the estimator by a Poisson process approximation. To our knowledge, local estimators are more robust to noisy data than global ones, but most of them generally underestimate $d$ when its value is sufficiently high. To address this problem few techniques have been proposed, among which the method described in [1] introduces a correction of the estimated intrinsic dimension based on the estimation of the errors obtained on synthetically produced datasets of known dimensionality.

In this work we present a local intrinsic dimension estimator, called IDEA, exploiting the statistical properties of manifold neighborhoods. Moreover, we compare our technique with state of the art algorithms. In Section 2 we describe our theoretical results, our base consistent estimator, and an asymptotic correction technique; in Section 3 experimental settings and results are reported; in Section 4 conclusions and future works are presented.

\section{The Algorithm}

In this section we present our theoretical results (see Section 2.1), our base algorithm (see Section 2.2), and an asymptotic estimation correction technique (see Section 2.3). 


\subsection{Theoretical Results}

Suppose to have a manifold $\mathcal{M} \equiv \mathcal{B}(\mathbf{0}, 1)$, where $\mathcal{B}(\mathbf{0}, 1)$ is a $d$-dimensional centered open ball with unitary radius, and choose $\psi$ as the identity map. To estimate the dimensionality $d$ of $\boldsymbol{B}(\mathbf{0}, 1)$ we need to identify a measurable characteristic of the hypersphere depending only on $d$. To achieve this goal, we consider that a $d$ dimensional vector randomly sampled from a $d$ dimensional hypersphere according to the uniform probability density function (pdf), can be generated by drawing a point $\hat{\boldsymbol{z}}$ from a standard normal distribution $\mathcal{N}(\cdot \mid \mathbf{0}, 1)$ and by scaling its norm (see Section 3.29 of [7]):

$$
z=\frac{u^{\frac{1}{d}}}{\|\hat{z}\|} \hat{z}, \quad \hat{z} \sim \mathcal{N}(\cdot \mid \mathbf{0}, 1)
$$

where $u$ is a random sample drawn from the uniform distribution $U(0,1)$.

Notice that, since $u$ is uniformly distributed, the quantities $1-u^{1 / d}$ are distributed according to the beta pdf $\beta_{1, d}$ with expectation $\mathbb{E}_{u \sim U(0,1)}\left[1-u^{1 / d}\right]=$ $\frac{1}{1+d}$. Therefore, the intrinsic dimensionality of the hypersphere is computed as:

$$
\mathbb{E}_{\boldsymbol{z} \sim \mathbf{B}_{d}}[1-\|\boldsymbol{z}\|]=\mathbb{E}_{\boldsymbol{z} \sim \mathbf{B}_{d}}\left[1-u^{\frac{1}{d}}\right]=\frac{1}{1+d} \Rightarrow d=\frac{\mathbb{E}_{\boldsymbol{z} \sim \mathbf{B}_{d}}[\|\boldsymbol{z}\|]}{1-\mathbb{E}_{\boldsymbol{z} \sim \mathbf{B}_{d}}[\|\boldsymbol{z}\|]}
$$

where $\mathbf{B}_{d}$ is the uniform pdf in the unit $d$-dimensional sphere. Notice that, embedding the hypersphere in a higher dimensional space $\Re^{D}$ by means of a map $\psi$ that applies only a rotation, does not change this result.

More generally, we now consider points uniformly drawn from a $d$-dimensional manifold $\mathcal{M} \equiv \Re^{d}$ embedded in $\Re^{D}$ through a smooth map $\psi: \mathcal{M} \rightarrow \Re^{D}$; under these assumptions their norms may be not distributed as $u^{\frac{1}{d}}$. Nevertheless, being $\psi$ a smooth map, close neighbors of $\mathcal{M}$ are mapped to close neighbors of $\Re^{D}$. Moreover, choosing a $d$-dimensional open ball $\mathcal{B}_{d}(\boldsymbol{c}, \epsilon)$ with center $\boldsymbol{c} \in \mathcal{M}$ and radius $\epsilon>0$, as long as $\psi$ preserves distances in $\mathcal{B}_{d}$, then for $\boldsymbol{z}$ uniformly drawn from $\boldsymbol{B}_{d}$, the distances $\frac{1}{\epsilon}\|\psi(\boldsymbol{c})-\psi(\boldsymbol{z})\|=\frac{1}{\epsilon}\|\boldsymbol{c}-\boldsymbol{z}\|$ are distributed as $u^{\frac{1}{d}}$, so that the result reported in Equation (2) is still valid and we obtain:

$$
d=\frac{\mathbb{E}_{\boldsymbol{z} \sim \mathbf{B}_{d}(\boldsymbol{c}, \epsilon)}\left[\frac{1}{\epsilon}\|\psi(\boldsymbol{c})-\psi(\boldsymbol{z})\|\right]}{1-\mathbb{E}_{\boldsymbol{z} \sim \mathbf{B}_{d}(\boldsymbol{c}, \epsilon)}\left[\frac{1}{\epsilon}\|\psi(\boldsymbol{c})-\psi(\boldsymbol{z})\|\right]}
$$

where, $\mathbf{B}_{d}(\boldsymbol{c}, \epsilon)$ is the uniform distribution in the ball $\boldsymbol{B}_{d}(\boldsymbol{c}, \epsilon)$.

To further generalize our theoretical results, we consider a locally isometric smooth map $\psi: \mathcal{M} \rightarrow \Re^{D}$, and samples drawn from $\mathcal{M} \equiv \Re^{d}$ by means of a non-uniform smooth pdf $f: \mathcal{M} \rightarrow \Re^{+}$. Notice that, being $\psi$ a local isometry, it induces a distance function $d_{\psi}(\cdot, \cdot)$ representing the metric on $\psi(\mathcal{M})$. Under these assumptions Equations (223) do not hold. However, without loss of generality, we consider $\boldsymbol{c}=\mathbf{0}_{d} \in \Re^{d}$ and $\psi(\boldsymbol{c})=\mathbf{0}_{D} \in \Re^{D}$, and we show that any smooth pdf $f$ is locally uniform where the probability is not zero. To this aim, assuming $f\left(\mathbf{0}_{d}\right)>0$ and $\boldsymbol{z} \in \Re^{d}$, we denote with $f_{\epsilon}$ the pdf obtained by setting $f_{\epsilon}(\boldsymbol{z})=0$ when $\|\boldsymbol{z}\|>1$, and $f_{\epsilon}(\boldsymbol{z}) \propto f(\epsilon \boldsymbol{z})$ when $\|\boldsymbol{z}\| \leq 1$. More precisely, denoting with $\chi_{\mathcal{B}_{d}(\mathbf{0}, 1)}$ the indicator function on the ball $\boldsymbol{\mathcal { B }}_{d}(\mathbf{0}, 1)$, we obtain:

$$
f_{\epsilon}(\boldsymbol{z})=\frac{f(\epsilon \boldsymbol{z}) \chi_{\boldsymbol{B}_{d}(\mathbf{0}, 1)}(\boldsymbol{z})}{\int_{\boldsymbol{t} \in \mathcal{B}_{d}(\mathbf{0}, 1)} f(\epsilon \boldsymbol{t}) d \boldsymbol{t}}
$$


Theorem 1. Given $\left\{\epsilon_{i}\right\} \rightarrow 0^{+}$, Equation (4) describes a sequence of $p d f$ having the unit d-dimensional ball as support; such sequence converges uniformly to the uniform distribution $\mathbf{B}_{d}$ in the ball $\boldsymbol{B}_{d}(\mathbf{0}, 1)$.

Proof. Evaluating the limit for $\epsilon \rightarrow 0^{+}$of the distance between $f_{\epsilon}$ and $\mathbf{B}_{d}$ in the supremum norm we get:

$$
\begin{aligned}
\lim _{\epsilon \rightarrow 0^{+}}\left\|f_{\epsilon}(\boldsymbol{z})-\mathbf{B}_{d}(\boldsymbol{z})\right\|_{\text {sup }} & =\lim _{\epsilon \rightarrow 0^{+}}\left\|\frac{f(\epsilon \boldsymbol{z}) \chi_{\mathcal{B}_{d}(\mathbf{0}, 1)}}{\int_{\mathcal{B}_{d}(\mathbf{0}, 1)} f(\epsilon \boldsymbol{t}) d \boldsymbol{t}}-\frac{\chi_{\mathcal{B}_{d}(\mathbf{0}, 1)}}{\int_{\mathcal{B}_{d}(\mathbf{0}, 1)} d \boldsymbol{t}}\right\|_{\text {sup }} \\
\{\text { just notation }\} & =\lim _{\epsilon \rightarrow 0^{+}}\left\|\frac{f(\epsilon \boldsymbol{z})}{\int_{\mathcal{B}_{d}(\mathbf{0}, 1)} f(\epsilon \boldsymbol{t}) d \boldsymbol{t}}-\frac{1}{\int_{\mathcal{B}_{d}(\mathbf{0}, 1)} d \boldsymbol{t}}\right\|_{\sup \boldsymbol{B}_{d}(\mathbf{0}, 1)} \\
\left\{\operatorname{setting} V=\int_{\mathcal{B}_{d}(0,1)} d \boldsymbol{t}\right\} & =\lim _{\epsilon \rightarrow 0^{+}}\left\|\frac{V f(\epsilon \boldsymbol{z})-\int_{\mathcal{B}_{d}(\mathbf{0}, 1)} f(\epsilon \boldsymbol{t}) d \boldsymbol{t}}{V \int_{\mathcal{B}_{d}(\mathbf{0}, 1)} f(\epsilon \boldsymbol{t}) d \boldsymbol{t}}\right\|\left\|_{\sup \mathcal{B}_{d}(\mathbf{0}, 1)}\right\| \int_{\mathcal{B}_{d}(\mathbf{0}, 1)} f(\epsilon \boldsymbol{t}) d \boldsymbol{t} \|_{\sup \mathcal{B}_{d}(\mathbf{0}, 1)}
\end{aligned}
$$

Defining:

$$
\min (\epsilon)=\min _{\mathcal{B}_{d}(\mathbf{0}, 1)} f(\epsilon \boldsymbol{z}) \quad \max (\epsilon)=\max _{\mathcal{B}_{d}(\mathbf{0}, 1)} f(\epsilon \boldsymbol{z})
$$

and noting that $\min (\epsilon)>0$ definitely since $f\left(\mathbf{0}_{d}\right)>0$, we have:

$$
\begin{aligned}
V \cdot \min (\epsilon) & \leq \quad V f(\epsilon \boldsymbol{z}) \quad \leq V \cdot \max (\epsilon) \\
V \cdot \min (\epsilon) & \leq \int_{\mathcal{B}_{d}(\mathbf{0}, 1)} f(\epsilon \boldsymbol{t}) d \boldsymbol{t} \leq V \cdot \max (\epsilon)
\end{aligned}
$$

thus their difference is bounded by $V(\max (\epsilon)-\min (\epsilon)) \underset{\epsilon \rightarrow 0^{+}}{\longrightarrow} 0^{+}$.

Theorem1 proves that the convergence of $f_{\epsilon}$ to $\mathbf{B}_{d}$ is uniform, so that in the limit $\left(\epsilon \rightarrow 0^{+}\right)$Equation (2) holds both for $d$-dimensional nonlinear manifolds embedded in $\Re^{D}$, and for points drawn by means of a non-uniform density function $f$. More precisely, for the smoothness and for the local isometry of $\psi$ :

$$
\mathbb{E}_{\boldsymbol{z} \sim f_{\epsilon}}\left[d_{\psi}\left(\psi(\boldsymbol{z}), \psi\left(\mathbf{0}_{d}\right)\right)\right]=\mathbb{E}_{\boldsymbol{z} \sim f_{\epsilon}}[\|\boldsymbol{z}\|] \underset{\epsilon \rightarrow 0^{+}}{\longrightarrow} \mathbb{E}_{\boldsymbol{z} \sim \mathbf{B}_{d}}[\|\boldsymbol{z}\|]=m
$$

\subsection{The Base Algorithm}

Consider a $d$-dimensional manifold $\mathcal{M} \equiv \Re^{d}$ non-linearly embedded in $\Re^{D}$ through a smooth locally isometric map $\psi: \mathcal{M} \rightarrow \Re^{D}$. Given a sample set $\boldsymbol{X}_{N}=\left\{\boldsymbol{x}_{i}\right\}_{i=1}^{N}=\left\{\psi\left(\boldsymbol{z}_{i}\right)\right\}_{i=1}^{N} \subset \Re^{D}$, where $\boldsymbol{z}_{i} \in \Re^{d}$ are independent identically distributed points drawn from $\mathcal{M}$ according to a smooth pdf $f: \mathcal{M} \rightarrow \Re^{+}$, our aim is to exploit our theoretical results to estimate the intrinsic dimensionality of $\mathcal{M}$ by means of the points in the set $\boldsymbol{X}_{N}$.

More precisely, the expectation of distances $\frac{1}{\epsilon} d_{\psi}(\psi(\boldsymbol{c}), \boldsymbol{x})$ for infinitesimal balls $\mathcal{B}_{D}(\psi(\boldsymbol{c}), \epsilon)$ with $\boldsymbol{c} \in \mathcal{M}$ must be estimated. To this aim, for each point 
$\boldsymbol{x}_{i} \in \boldsymbol{X}_{N}$ we find the set of $k+1(1 \leq k \leq N-1)$ nearest neighbors $\hat{\boldsymbol{X}}_{k+1}^{N}=$ $\hat{\boldsymbol{X}}_{k+1}^{N}\left(\boldsymbol{x}_{i}\right)=\left\{\boldsymbol{x}_{j}\right\}_{j=1}^{k+1} \subset \boldsymbol{X}_{N}$. Call $\hat{\boldsymbol{x}}=\hat{\boldsymbol{x}}_{k+1}^{N}\left(\boldsymbol{x}_{i}\right) \in \hat{\boldsymbol{X}}_{k+1}^{N}$ the most distant point from $\boldsymbol{x}_{i}$, and denote $\boldsymbol{X}_{k}^{N}=\boldsymbol{X}_{k}^{N}\left(\boldsymbol{x}_{i}\right)=\hat{\boldsymbol{X}}_{k+1}^{N} \backslash\{\hat{\boldsymbol{x}}\}$. Notice that, when $\boldsymbol{x}_{i}$ is fixed, almost surely (a.s.) we have $\left\|\boldsymbol{x}-\boldsymbol{x}_{i}\right\|<\left\|\hat{\boldsymbol{x}}-\boldsymbol{x}_{i}\right\| \forall \boldsymbol{x} \in \boldsymbol{X}_{k}^{N}$; therefore, we can consider points in $\boldsymbol{X}_{k}^{N}$ as drawn from the open ball $\boldsymbol{B}_{D}\left(\boldsymbol{x}_{i},\left\|\hat{\boldsymbol{x}}-\boldsymbol{x}_{i}\right\|\right)$. Exploiting this fact, in order to estimate the intrinsic dimension $d$ of $\mathcal{M}$, we estimate the expectation of distances as follows:

$$
m \simeq \frac{1}{k} \sum_{\boldsymbol{x} \in \boldsymbol{X}_{k}^{N}} \frac{\left\|\boldsymbol{x}_{i}-\boldsymbol{x}\right\|}{\|\hat{\boldsymbol{x}}-\boldsymbol{x}\|}
$$

Note that $m$ depends only upon the intrinsic dimensionality $d$ of $\mathcal{M}$ and does not depend on the chosen center $\boldsymbol{x}_{i}$.

Corollary 1. Given two sequences $\left\{k_{j}\right\}$ and $\left\{N_{j}\right\}$ such that for $j \rightarrow+\infty$ :

We have the limit:

$$
k_{j} \rightarrow+\infty, \quad N_{j} \rightarrow+\infty, \quad \frac{k_{j}}{N_{j}} \rightarrow 0
$$

$$
\lim _{j \rightarrow+\infty} \frac{1}{k_{j}} \sum_{\boldsymbol{x} \in \boldsymbol{X}_{k_{j}}^{N_{j}}} \frac{\left\|\boldsymbol{x}_{i}-\boldsymbol{x}\right\|}{\|\hat{\boldsymbol{x}}-\boldsymbol{x}\|}=m \quad \text { a.s. }
$$

Proof. Considering the sequences $\left\{k_{j}\right\}$ and $\left\{N_{j}\right\}$, the conditions reported in Equation (6) ensure that $\epsilon=\left\|\hat{\boldsymbol{x}}-\boldsymbol{x}_{i}\right\| \rightarrow 0^{+}$when $j \rightarrow+\infty \mathbf{1}^{1}$. Theorem 4 in [4] ensures that geodetic distances in the infinitesimal ball converge to Euclidean distances with probability 1 ; furthermore, the sample mean is an unbiased estimator for the expectation (law of large numbers); moreover, Theorem 1 guarantees that the underlying pdf converges to the uniform one. We have:

$$
\begin{aligned}
\lim _{j \rightarrow+\infty} \frac{1}{k_{j}} \sum_{\boldsymbol{x} \in \boldsymbol{X}_{k_{j}}^{N_{j}}} \frac{\left\|\boldsymbol{x}_{i}-\boldsymbol{x}\right\|}{\|\hat{\boldsymbol{x}}-\boldsymbol{x}\|} & =\lim _{j \rightarrow+\infty} \frac{1}{k_{j} \epsilon} \sum_{\boldsymbol{x} \in \boldsymbol{X}_{k_{j}}^{N_{j}}} d_{\psi}\left(\boldsymbol{x}_{i}, \boldsymbol{x}\right)+o(\epsilon)= \\
\lim _{\epsilon \rightarrow 0^{+}} \mathbb{E}_{\boldsymbol{z} \sim f_{\epsilon}}\left[\frac{1}{\epsilon} d_{\psi}\left(\psi(\boldsymbol{z}), \psi\left(\mathbf{0}_{d}\right)\right)\right] & =\lim _{\epsilon \rightarrow 0^{+}} \mathbb{E}_{\boldsymbol{z} \sim \mathbf{B}_{d}(\mathbf{0}, \epsilon)}\left[\frac{1}{\epsilon} d_{\psi}\left(\psi(\boldsymbol{z}), \psi\left(\mathbf{0}_{d}\right)\right)\right]= \\
\lim _{\epsilon \rightarrow 0^{+}} \frac{1}{\epsilon} \epsilon \mathbb{E}_{\boldsymbol{z} \sim \mathbf{B}_{d}(\mathbf{0}, 1)}\left[d_{\psi}\left(\psi(\boldsymbol{z}), \psi\left(\mathbf{0}_{d}\right)\right)\right] & =\mathbb{E}_{\boldsymbol{z} \sim \mathbf{B}_{d}(\mathbf{0}, 1)}[\|\boldsymbol{z}\|]=m
\end{aligned}
$$

By employing Equation (2) and Corollary 1, we get a consistent estimator $\hat{d}$ for the intrinsic dimensionality $d$ of $\mathcal{M}$ as follows:

$$
\begin{aligned}
& m \simeq \hat{m}=\frac{1}{N k} \sum_{i=1}^{N} \sum_{\boldsymbol{x} \in \boldsymbol{X}_{k}^{N}} \frac{\left\|\boldsymbol{x}_{i}-\boldsymbol{x}\right\|}{\|\hat{\boldsymbol{x}}-\boldsymbol{x}\|} \\
& d=\frac{m}{1-m} \simeq \frac{\hat{m}}{1-\hat{m}}=\hat{d}
\end{aligned}
$$

\footnotetext{
${ }^{1}$ See proof of Theorem 4 in [4] where $k$ must be substituted by $o(n)$.
} 


\subsection{Asymptotic Correction}

Although the algorithm described in Section 2.2 proposes a consistent estimator of the intrinsic dimension, when the dimensionality is too high, the number of sample points becomes insufficient to compute an acceptable estimation. This is due to the fact that, as shown in [6], the number of sample points required to perform dimensionality estimation with acceptable results, grows exponentially with the value of the intrinsic dimensionality ("curse of dimensionality").

In literature, some methods have been proposed to reduce this effect. For instance, in [1] the authors propose a correction of the estimated intrinsic dimension based on the estimation of the errors obtained on synthetically produced datasets of known dimensionality (hypercubes). Another interesting approach has been proposed in [3], where the authors propose a non parametric least square strategy based on re-sampling from the point population $\boldsymbol{X}_{N}$.

Similarly, in our work we propose a method that allows to study the asymptotic behavior described by the available data. To this aim, we adopt a Monte Carlo approach performing $R$ runs of the algorithm reported in Section 2.2. We extract from the given dataset $\boldsymbol{X}_{N}$ random subsets $\boldsymbol{\mathcal { R }}_{r=1}^{R}$ with different cardinalities $\boldsymbol{R}_{r=1}^{R}$. The cardinalities $\boldsymbol{R}_{r}$ are randomly generated by means of the binomial distribution $\operatorname{Binom}(N, p)$, where the value of $p$ spans a fixed rang 2 . The intrinsic dimensionality, estimated during each run, becomes a sample from a "trend curve"; moreover, for each subsample we choose the kNN parameter $k_{r}=\lceil k \sqrt{p}\rceil$, trying to emulate a sequence $\left\{k_{r}\right\}$ such that $k_{r} \rightarrow+\infty, \boldsymbol{R}_{r} \rightarrow+\infty$, and $\frac{k_{r}}{R_{r}} \rightarrow 0$, thus fulfilling the conditions reported in Equation (6).

We noticed that, when the base algorithm proposed in Section 2.2 underestimates the intrinsic dimensionality, its application to point subsets $\mathcal{R}_{r=1}^{R}$ with increasing cardinality produces increasing estimations of the intrinsic dimension $\hat{d}=\hat{d}\left(\boldsymbol{R}_{r}\right)$. As demonstrated in Section 2.2, these estimates converge to the real intrinsic dimensionality for $j \rightarrow+\infty$ (see conditions reported in Equation (6)). Our assumption, based on this empirical observation, is that the function $\hat{d}(N)$ has a horizontal asymptote. Therefore, we fit the pairs $\left(\log \left(\boldsymbol{R}_{r}\right), \hat{d}\left(\boldsymbol{R}_{r}\right)\right)$ by means of the parametric function 3 described below:

$$
\hat{d}\left(\boldsymbol{R}_{r}\right) \simeq g\left(\boldsymbol{R}_{r}\right)=a_{0}-\frac{a_{1}}{\log _{2}\left(\frac{\boldsymbol{R}_{r}}{a_{2}}+a_{3}\right)}
$$

where $\left\{a_{i}\right\}_{i=0}^{3}$ are fitting parameters controlling translation and scaling on both axes; their values are computed by a non-linear least squares fitting algorithm. Notice that, since $\lim _{\boldsymbol{R}_{r} \rightarrow+\infty} g\left(\boldsymbol{R}_{r}\right)=a_{0}$ then the asymptote of Equation (10) is $\hat{d}=a_{0}$. Moreover, the derivate $g^{\prime}=D\left[g\left(\boldsymbol{R}_{r}\right)\right]$ shows that the parameter $a_{1}$ controls the increasing/decreasing behavior of the function $g$. For these reasons, when the estimated parameter $a_{1}>0$ (increasing function), we use the

\footnotetext{
${ }^{2}$ In our tests $p \in\{0.1, \cdots, 0.9\}$.

3 The choice of using 2 as the log base does not affect the results, being the change of base just a change of scale in the $y$ axis.
} 
Table 1. Brief description of the synthetic and real datasets, where $d$ is the intrinsic dimension, and $D$ is the embedding space dimension. In the second column of the synthetic data, the number in the subscript refers to the dataset name used by the generator proposed in 11.

\begin{tabular}{|c|c|c|c|l|}
\hline Dataset & Name & $\boldsymbol{d}$ & $\boldsymbol{D}$ & Description \\
\hline \hline \multirow{5}{*}{ Syntethic } & $\mathcal{M}_{1}$ & 10 & 11 & Uniformly sampled sphere linearly embedded. \\
& $\mathcal{M}_{2}$ & 3 & 5 & Affine space. \\
& $\mathcal{M}_{3}$ & 4 & 6 & Concentrated figure, easy to confuse with a 3d one. \\
& $\mathcal{M}_{4}$ & 4 & 8 & Non-linear manifold. \\
& $\mathcal{M}_{5}$ & 2 & 3 & 2-d Helix \\
& $\mathcal{M}_{6}$ & 6 & 36 & Non-linear manifold. \\
& $\mathcal{M}_{7}$ & 2 & 3 & Swiss-Roll. \\
& $\mathcal{M}_{8}$ & 12 & 72 & Non-linear manifold. \\
& $\mathcal{M}_{10 a}$ & 20 & 20 & Affine space. \\
& $\mathcal{M}_{10 b}$ & 10 & 11 & Uniformly sampled hypercube. \\
& $\mathcal{M}_{10 c}$ & 24 & 18 & Uniformly sampled hypercube. \\
& $\mathcal{M}_{11}$ & 2 & 3 & Uniformly sampled hypercube. \\
& $\mathcal{M}_{12}$ & 20 & 20 & Möebius band 10-times twisted. \\
& $\mathcal{M}_{13}$ & 1 & 13 & Curve. \\
\hline \hline \multirow{5}{*}{ Real } & $\mathcal{M}_{\text {Faces }}$ & 3 & 4096 & ISOMAP face dataset. \\
& $\mathcal{M}_{\text {MNIST1 }}$ & $8-11$ & 784 & MNIST database (digit 1). \\
& $\mathcal{M}_{M N I S T 3}$ & $12-14$ & 784 & MNIST database (digit 3). \\
\hline
\end{tabular}

parameter $a_{0}$ as the final estimate for $d$; otherwise, we use the estimation obtained by the base algorithm applied to the whole dataset.

To obtain a stable estimation of the intrinsic dimension we execute the asymptotic correction algorithm 20 times and we average the obtained results.

\section{Algorithm Evaluation}

In this section we describe the datasets employed in our experiments (see Section 3.1 ), we summarize the adopted experimental settings (see Section 3.2), and we report the achieved results (see Section 3.3).

\subsection{Dataset Description}

To evaluate our algorithm, we have performed experiments on both synthetic and real datasets (see Table 1). To generate the synthetic datasets we have employed the tool proposed in [1]. The real datasets are the ISOMAP face database [18] and the MNIST database 14. The ISOMAP face dataset consists in 698 graylevel images of size $64 \times 64$ depicting the face of a sculpture. This dataset has three degrees of freedom: two for the pose and one for the lighting direction. The MNIST database consists in 70000 gray-level images of size $28 \times 28$ of handwritten digits; in our tests we have used the 6742 training points representing 
Table 2. Parameter settings for the different estimators: $k$ represents the number of neighbors, $\gamma$ the edge weighting factor for kNN, $M$ the number of Least Square (LS) runs, and $N$ the number of resampling trials per LS iterations

\begin{tabular}{|c|c|c|}
\hline Method & Synthetic & Real \\
\hline \hline PCA & Threshold $=0.025$ & Threshold $=0.0025$ \\
CD & None & None \\
MLE & $k_{1}=6 k_{2}=20$ & $k_{1}=3 k_{2}=8$ \\
$\mathrm{kNNG}_{1}$ & $k_{1}=6, k_{2}=20, \gamma=1, M=1, N=10$ & $k_{1}=3, k_{2}=8, \gamma=1, M=1, N=10$ \\
$\mathrm{kNNG}_{2}$ & $k_{1}=6, k_{2}=20, \gamma=1, M=10, N=1$ & $k_{1}=3, k_{2}=8, \gamma=1, M=10, N=1$ \\
IDEA & $k=20$ & $k=8$ \\
\hline
\end{tabular}

the digit 1 and the 6131 training points representing the digit 3 . The intrinsic dimension of the datasets extracted from the MNIST database is not actually known, but some works have proposed similar estimations [114] for the different digits. More precisely, these works report an estimation in the range $d \in\{8 . .11\}$ for digit 1, and between 12 and 14 for the digit 3 .

\subsection{Experimental Setting}

We have compared our method with well-known global (PCA, kNNG) and local (CD, MLE) intrinsic dimension estimators. For kNNG and MLE4, we have used the authors' Matlab implementation, and we have employed the version in the toolbox of dimensionality reduction for the other algorithms. 5 .

We have used the synthetic dataset generator [11] to create 20 instances of each dataset reported in Table 1, each of which was composed by 2000 randomly sampled points. To execute multiple tests on $\boldsymbol{M}_{M N I S T 1}$ and $\boldsymbol{M}_{M N I S T 3}$, we have extracted 5 random subsets per dataset containing 2000 points each. To obtain a stable estimation, for each technique we have averaged the results achieved on the different subsets. Table 2 summarizes the employed configuration parameters. To relax the dependency of kNNG from the selection of $k$, we performed multiple runs with $k_{1} \leq k \leq k_{2}$ and we averaged the achieved results.

\subsection{Experimental Results}

In this subsection results achieved on both real and synthetic datasets are reported. On synthetic datasets (see Table 3) our method has computed good approximations both on low and high intrinsic dimensional datasets, achieving results always comparable with those that better approximate the real intrinsic dimension. We further note that IDEA has generally outperformed global methods on strongly non-linearly embedded manifolds obtaining good estimations on

\footnotetext{
${ }^{4}$ http://www.eecs.umich.edu/ hero/IntrinsicDim/, http://www.stat.lsa.umich.edu/ elevina/mledim.m

${ }^{5}$ http://cseweb.ucsd.edu/ lvdmaaten/dr/download.php
} 
Table 3. Results achieved on synthetic datasets by global and local approaches. In bold the best approximations achieved both by global and by local techniques.

\begin{tabular}{|c|c||c|c|c||c|c|c|}
\hline Dataset & Int. Dim & PCA & kNNG $_{1}$ & $\mathrm{kNNG}_{2}$ & CD & MLE & IDEA \\
\hline \hline $\mathcal{M}_{13}$ & 1 & 4 & $\mathbf{1 . 0 0}$ & 1.01 & 1.07 & $\mathbf{1 . 0 0}$ & 1.12 \\
$\mathcal{M}_{5}$ & 2 & 3 & 1.96 & $\mathbf{2 . 0 0}$ & $\mathbf{1 . 9 8}$ & 1.96 & 2.07 \\
$\mathcal{M}_{7}$ & 2 & 3 & 1.93 & $\mathbf{1 . 9 8}$ & 1.94 & $\mathbf{1 . 9 7}$ & $\mathbf{1 . 9 7}$ \\
$\mathcal{M}_{11}$ & 2 & 3 & 1.96 & $\mathbf{2 . 0 1}$ & 2.23 & 2.30 & $\mathbf{1 . 9 8}$ \\
$\mathcal{M}_{2}$ & 3 & $\mathbf{3}$ & 2.85 & 2.93 & 2.88 & 2.87 & $\mathbf{3 . 0 7}$ \\
$\mathcal{M}_{3}$ & 4 & $\mathbf{4}$ & 3.80 & 4.22 & 3.16 & 3.82 & $\mathbf{4 . 0 1}$ \\
$\mathcal{M}_{4}$ & 4 & 8 & 4.08 & $\mathbf{4 . 0 6}$ & 3.85 & $\mathbf{3 . 9 8}$ & 4.07 \\
$\mathcal{M}_{6}$ & 6 & 12 & $\mathbf{6 . 5 3}$ & 13.99 & $\mathbf{5 . 9 1}$ & 6.45 & 6.79 \\
$\mathcal{M}_{1}$ & 10 & 11 & 9.07 & $\mathbf{9 . 3 9}$ & 9.09 & 9.06 & $\mathbf{1 0 . 3 5}$ \\
$\mathcal{M}_{10 a}$ & 10 & $\mathbf{1 0}$ & 8.35 & 9.00 & 8.04 & 8.22 & $\mathbf{1 0 . 0 7}$ \\
$\mathcal{M}_{8}$ & 12 & 24 & $\mathbf{1 4 . 1 9}$ & 8.29 & 10.91 & $\mathbf{1 3 . 6 9}$ & 14.45 \\
$\mathcal{M}_{10 b}$ & 17 & $\mathbf{1 7}$ & 12.85 & 15.58 & 12.09 & 12.77 & $\mathbf{1 6 . 7 8}$ \\
$\mathcal{M}_{9}$ & 20 & $\mathbf{2 0}$ & 14.87 & 17.07 & 13.60 & 14.54 & $\mathbf{1 6 . 8 1}$ \\
$\mathcal{M}_{12}$ & 20 & $\mathbf{2 0}$ & 16.50 & 14.58 & 11.24 & 15.67 & $\mathbf{2 1 . 0 8}$ \\
$\mathcal{M}_{10 c}$ & 24 & $\mathbf{2 4}$ & 17.26 & 23.68 & 15.48 & 16.80 & $\mathbf{2 3 . 9 4}$ \\
\hline
\end{tabular}

Table 4. Results achieved on real datasets by global and local approaches. In bold the best approximations achieved both by global and by local techniques.

\begin{tabular}{|c|c||c|c|c||c|c|c|}
\hline Dataset & Int. Dim & PCA & kNNG $_{1}$ & kNNG $_{2}$ & CD & MLE & IDEA \\
\hline \hline $\mathcal{M}_{\text {Faces }}$ & 3 & 21 & $\mathbf{3 . 6 0}$ & 4.32 & $\mathbf{3 . 3 7}$ & 4.05 & 3.73 \\
$\mathcal{M}_{\text {MNIST } 1}$ & $8-11$ & 11.80 & 10.37 & $\mathbf{9 . 5 8}$ & 6.96 & $\mathbf{1 0 . 2 9}$ & 11.06 \\
$\mathcal{M}_{\text {MNIST3 }}$ & $12-14$ & 9.80 & 37.70 & $\mathbf{1 6 . 1 6}$ & 10.16 & 15.67 & $\mathbf{1 4 . 9 8}$ \\
\hline
\end{tabular}

high intrinsic dimensional datasets where local techniques have usually underestimated the correct value. These behaviors have confirmed the quality of the asymptotic correction algorithm.

In Table 4 the results achieved on real datasets have been summarized; notice that, using real data that are often noisy, IDEA has obtained either the best approximation of the intrinsic dimension or stable results comparable with those achieved by the best performing technique.

\section{Conclusions and Future Works}

In this work we have presented a consistent local intrinsic dimension estimator that exploits the statistical properties of manifold neighborhoods. Moreover, we have proposed an asymptotic correction of our algorithm to reduce the underestimate behavior that affects most of the local intrinsic dimension estimation methods. The promising results achieved, and the comparison with state of the art techniques, have confirmed the quality of the proposed technique. Moreover, in our tests, only IDEA has obtained good estimations both on low and high intrinsic dimensional datasets, and both on linear and non-linear embeddings. 
In future works we want to focus on the asymptotic correction algorithm to improve the theoretical background about the fitting function.

\section{References}

1. Camastra, F., Vinciarelli, A.: Estimating the intrinsic dimension of data with a fractal-based method. IEEE Trans. PAMI 24, 1404-1407 (2002)

2. Costa, J.A., Hero, A.O.: Geodesic entropic graphs for dimension and entropy estimation in manifold learning. IEEE Trans. on SP 52(8), 2210-2221 (2004)

3. Costa, J.A., Hero, A.O.: Learning intrinsic dimension and entropy of highdimensional shape spaces. In: EUSIPCO (2004)

4. Costa, J.A., Hero, A.O.: Learning intrinsic dimension and entropy of shapes. In: Krim, H., Yezzi, T. (eds.) Statistics and Analysis of Shapes. Birkhäuser, Basel (2005)

5. Donoho, D.L., Grimes, C.: Hessian Eigenmaps: New Locally Linear Embedding Techniques For High-Dimensional Data. Technical report (July 2003)

6. Eckmann, J.P., Ruelle, D.: Fundamental limitations for estimating dimensions and lyapunov exponents in dynamical systems. Physica D 56(2-3), 185-187 (1992)

7. Fishman, G.S.: Monte Carlo: Concepts, Algorithms, and Applications. Springer Series in Operations Research. Springer, New York (1996)

8. Fukunaga, K.: Intrinsic Dimensionality Extraction. Classification. In: Pattern Recognition and Reduction of Dimensionality (1982)

9. Grassberger, P., Procaccia, I.: Measuring the strangeness of strange attractors. Physica D: Nonlinear Phenomena 9, 189 (1983)

10. Gupta, M.D., Huang, T.: Regularized maximum likelihood for intrinsic dimension estimation. In: UAI (2010)

11. Hein, M.: Intrinsic dimensionality estimation of submanifolds in euclidean space. In: ICML, pp. 289-296 (2005)

12. Jollife, I.T.: Principal Component Analysis. Springer Series in Statistics. Springer, New York (1986)

13. Kégl, B.: Intrinsic dimension estimation using packing numbers. In: Proc. of NIPS, pp. 681-688 (2002)

14. LeCun, Y., Cortes, C.: Gradient-Based Learning Applied to Document Recognition. Proceedings of the IEEE 86(11), 2278-2324 (1998)

15. Levina, E., Bickel, P.J.: Maximum likelihood estimation of intrinsic dimension. In: NIPS, vol. 1, pp. 777-784 (2005)

16. Mordohai, P., Medioni, G.: Dimensionality estimation, manifold learning and function approximation using tensor voting. J. Mach. Learn. Res. 11, 411-450 (2010)

17. Roweis, S.T., Saul, L.K.: Nonlinear Dimensionality Reduction by Locally Linear Embedding. Science 290, 2323-2326 (2000)

18. Tenenbaum, J.B., Silva, V., Langford, J.C.: A Global Geometric Framework for Nonlinear Dimensionality Reduction. Science 290, 2319-2323 (2000) 